\title{
ESTUDIO DE ACCESIBILIDAD PEATONAL DE LA ZONA ENTRE LAS ESTACIONES DEL METRO DE PANAMÁ DE SANTO TOMÁS Y LOTERÍA
}

\author{
Mosquera, Alfonso \\ Grupo de Investigación del Transporte y Territorio, Facultad de Ingeniería. \\ Civil, Universidad Tecnológica de Panamá. \\ Ciudad de Panamá, Panamá. \\ alfonso.mosquera@utp.ac.pa. \\ Bethancourt, Gabriel \\ Grupo de Investigación del Transporte y Territorio, Facultad de Ingeniería. \\ Civil, Universidad Tecnológica de Panamá. \\ Ciudad de Panamá, Panamá. \\ gabriel.bethancourt@utp.ac.pa. \\ Quijada-Alarcón, Jorge \\ Grupo de Investigación del Transporte y Territorio, Facultad de Ingeniería. \\ Civil, Universidad Tecnológica de Panamá. \\ Ciudad de Panamá, Panamá. \\ jorge.quijada@utp.ac.pa.
}

\section{ABSTRACT}

The sidewalks of Panama City do not consider providing ease and security to people with or without reduced mobility. Hasty development, population density, limits pedestrian accessibility. It seeks to improve the existing structure, being an area of interest, the pedestrian network surrounding the metro. Space is limited and mostly allocated to vehicles. The main objective was to analyze the sidewalks of the area taking as a main reference the parameters established by SENADIS on the sidewalk width required for optimal transit and with this, to determine favorable routes. The collection of structural information and accessibility status on the sidewalks through field research sought to present and visually manage the behavior on mobility and accessibility in the area. Bearing in mind that it is necessary to improve the level of accessibility in the existing structure, as well as to distinguish the segments according to the accessibility they possess. The methodology consisted of collecting the existing information and evaluating the accessibility status according to previously established variablessuch as: minimum width of sidewalks, 
structural condition, obstacles, sanitation, signs and ramps. Results were obtained such as the number of optimal, functional and deficient sections. Thus, as the sections that meet the width of accessibility, were also presented the sections that house sewage, garbage, and obstacles. In this way, maps were created that allow to see the state of the sidewalks in three categories (optimal, functional and deficient) mainly.

Keywords: sidewalks, mobility, security, accessibility, maps.

\section{RESUMEN}

Las aceras de la Ciudad de Panamá no consideran que se brinde facilidad y seguridad a personas con o sin movilidad reducida. El desarrollo apresurado, densidad poblacional, limita la accesibilidad peatonal. Se busca mejorar la estructura existente, siendo zona de interés la red peatonal circundante al metro. El espacio es reducido y en su mayor parte asignado a los vehículos. El objetivo principal fue analizar las aceras de la zona tomando como referencia principal los parámetros establecidos por el SENADIS sobre al ancho de acera requerido para un tránsito óptimo y con esto, determinar rutas favorables. La recopilación de la información estructural y el estado de accesibilidad en las aceras a través de la investigación de campo buscó presentar y gestionar de forma visual el comportamiento sobre la movilidad y accesibilidad en la zona. Teniendo presente que se requiere mejorar el nivel de accesibilidad en la estructura existente, así como distinguir los segmentos en función de la accesibilidad que poseen. La metodología consistió en recopilar la información existente y evaluar el estado de accesibilidad conforme a variables previamente establecidas como: ancho mínimo de aceras, estado estructural, obstáculos, salubridad, señalizaciones y rampas. Se obtuvieron resultados tales como la cantidad de tramos óptimos, funcionales y deficientes. Así, como los tramos que cumplencon el ancho de accesibilidad, también se presentaron los tramos que albergan aguas negras, basuray obstáculos. De esta forma, se crearon mapas que permiten ver el estado de las aceras en tres categorías (óptimas, funcionales y deficientes) principalmente.

Palabras claves: aceras, movilidad, seguridad, accesibilidad, mapas.

\section{INTRODUCCIÓN.}

La gran generación de viajes que se presenta a diario en el centro de la Ciudad de Panamá produce un aglomeramiento de personas en los puntos donde no se ha gestionado de 
manera correcta la circulación y accesibilidad peatonal. Debido a esto, se genera el interés por representar de forma precisa cada una de estas deficiencias, en pro de proveer una solución funcional y sostenible, por medio de la generación de ciertos indicadores que pueden permitir la toma de decisión de las entidades responsables del mantenimiento y estructuración de la forma en cómo se movilizan las personas.

La recopilación de la información estructural y el estado de accesibilidad en las aceras a través de la investigación de campo busca presentar y gestionar de forma visual a través de herramientas geográficas (SIG) [1]. El comportamiento actual sobre la movilidad y accesibilidad en la zona de estudio, buscando mejorar la planificación enfocada a las personas con movilidad reducida [1]. Teniendo presente que el estudio busca elevar el nivel de la accesibilidad en la estructura existente, al distinguir los segmentos en función de la accesibilidad que poseen al evaluarse determinadas variables, convirtiéndose en un modelo base o comparativo para investigaciones relacionadas con la accesibilidad peatonal universal.

\section{MÉTODO}

\section{A. Esquema de recorrido}

Para efectuar de forma ordenada y simplificada el levantamiento de la información en la zona de estudio, se realizó un esquema de recorrido a través de la herramienta Google Earth, con el fin de obtener una idea de las cuadras que componen el área de estudio. Preliminarmente se utilizaron las estaciones del metro de Lotería y Santo Tomás como puntos de interés.

\section{B. Captación y representación de los datos}

La captación de los datos se realizó principalmente por medio de apuntes, registro de coordenadascon la aplicación Mobile Topographer y Google Earth.

Con la información levantada, registrada y cotejada, se procesó mediante ArcGIS PRO para realizar los análisis definidos en los parámetros de la investigación, así como cualquier otro resultado que fuese de utilidad. El software ArcGIS Pro, fue la herramienta utilizada para el procesamiento y representación geográfica espacial de la información obtenida en campo. Las diferentes herramientas de geoprocesamiento y manejo espacial de ArcGIS PRO, permite enlazar los datos mediante la utilización de atributos en entidades visuales que pueden representar el comportamiento de dichos datos [2].

De forma simplificada, estos datos de campo se establecieron en un archivo tipo XML, en donde las variables y datos fueron organizados en función del orden del levantamiento 
efectuado. Luego de acoplar los datos, se utilizan las herramientas de análisis vectorial (puntos, líneas y polígonos). A los cuales se les pueden asignar los valores de las variables que puedan representar. Para el análisis del estado de las aceras se utilizaron las 3 primeras categorías de las variables de campo (escena urbana, seguridad y estructura). Con el fin de generar un Mapa que muestre la condición que se encontró en cada uno de los tramos.

\section{RESULTADOS}

En la zona de estudio se levantaron 100 tramos con infraestructura peatonal existente y 14 tramos extras donde se observó tránsito de peatones sobre espacios sin infraestructura peatonal. Para la estadística y evaluación general se utilizaron los 100 tramos con infraestructura existente. Por el contrario, para la estadística sobre accesibilidad global de la zona se utilizaron los 114 tramos.

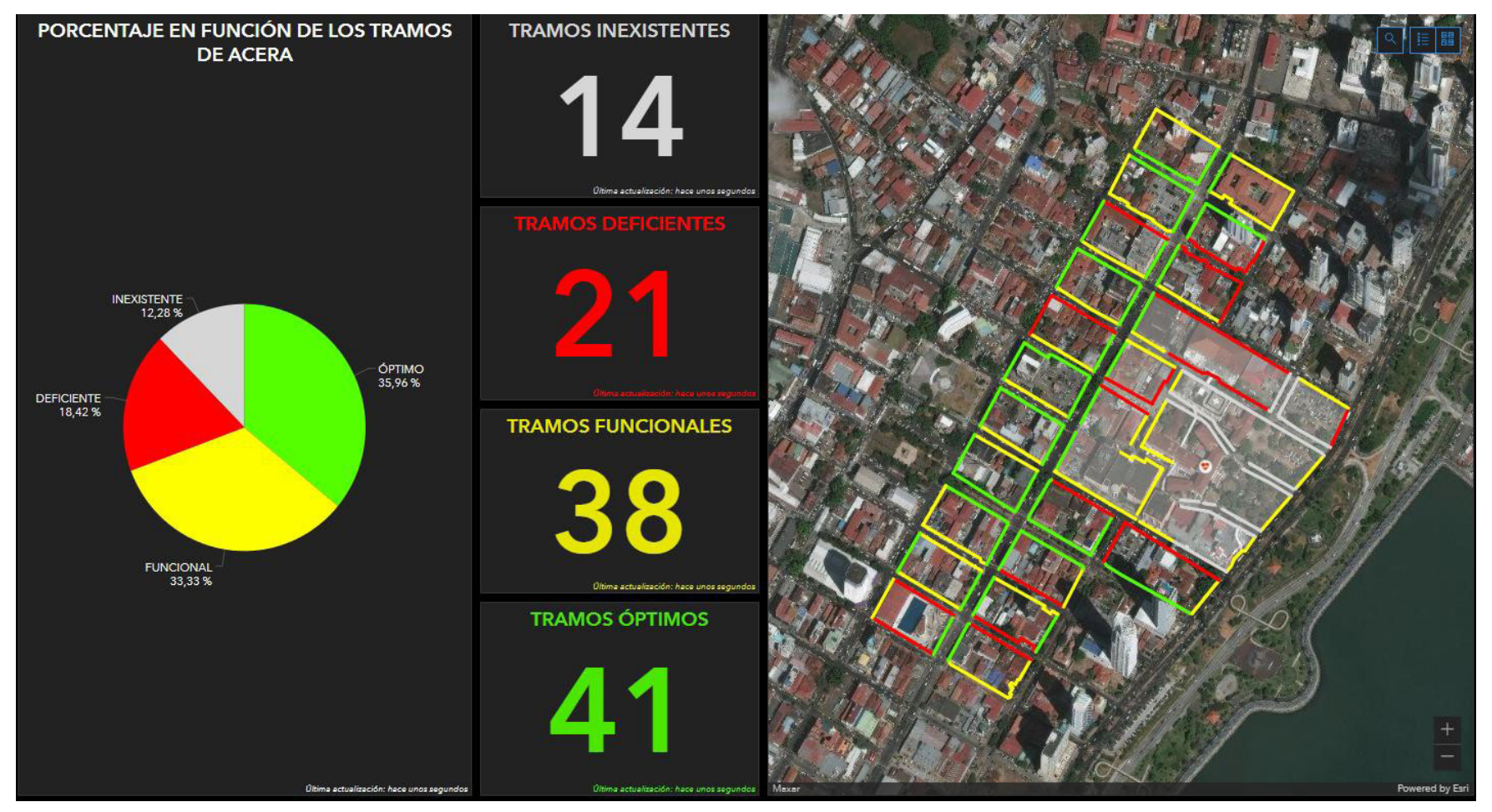

Clasificación de los tramos dentro de la zona de estudio.

\begin{tabular}{|c|c|c|}
\hline Tramos de la zona de estudio & Cantidad & Tramos de la zona de estudio \\
\hline Óptimos & 41 & Optimos \\
\hline Funcionales & 38 & Funcionales \\
\hline Deficientes & 21 & Deficientes \\
\hline Inexistentes & 14 & Inexistentes \\
\hline
\end{tabular}


En primera instancia del análisis se puede inferir que la zona se encuentra en estado funcional para circular al tener un $69.29 \%$ de accesibilidad peatonal que resulta de la suma de los tramos óptimos y funcionales en base a los tramos totales, no obstante, en un análisis más detallado de la zona, se puede apreciar que uno de los puntos de interés más relevantes del estudio como lo es la zona hospitalaria, en la que se ubican tanto el Hospital Santo Tomás como el Hospital del Niño, seencuentra con apenas un $47.4 \%$ de accesibilidad con 38 tramos totales, 4 tramos óptimos, 14 tramos funcionales, 6 tramos deficientes y 14 tramos inexistentes lo cual es de resaltar ya que al ser una zonacon tanto flujo de peatones que se dirigen en busca de atención médica, se esperaría que las acerasde esta zona al menos lleguen a ser funcionales para que transiten por ellas.

Otro hecho importante a resaltar es que las aceras que se encuentran en medio de las salidas de las estaciones del Metro de Panamá, todas se encuentran en un estado óptimo. Además, existen 5 tramosque cumplen con todas las variables del análisis, por lo tanto, tienen un índice de 1 , de estos 2 son pertenecientes a los tramos que se encuentran en las salidas de las estaciones del metro.

\section{CONCLUSIONES}

Para el análisis del estado de las aceras se puede concluir que, según los resultados obtenidos, la zona se encuentra de manera general en un estado funcional para el tránsito de personas sinproblemas de movilidad. No obstante, para la zona hospitalaria se puede aseverar que se encuentra en un estado deficiente.

Para observar los resultados extendidos de esta investigación, puede acceder mediante el siguiente código QR mostrado en la imagen.

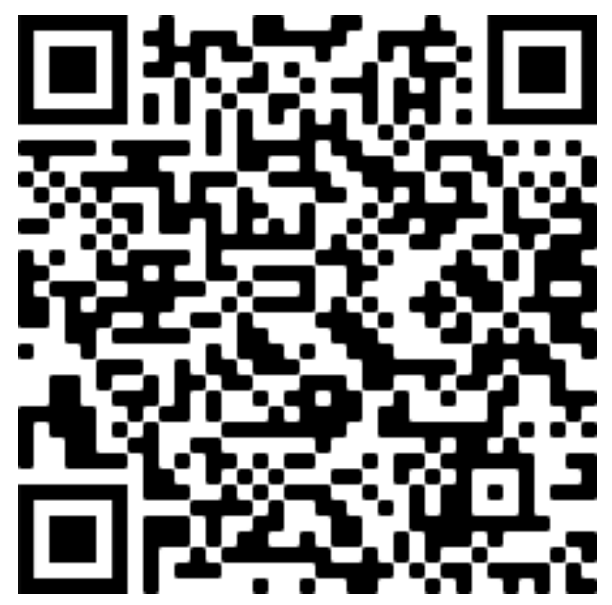




\section{REFERENCIAS}

[1] ESRI, «ArcGIS Resources,» 101 2021. [En línea]. Available: https://resources.arcgis.com/es/help/ getting-started/articles/026n0000000t000000.htm.

[2] Esri The Sciencie Of Where, «ArcGIS Pro,» 121 2021. [En línea]. Available: https://pro.arcgis.com/ es/pro-app/help/analysis/networks/service-area-analysis-layer.htm\#.

\section{AUTORIZACIÓN Y LICENCIA CC}

Los autores autorizan a APANAC XVIII a publicar el artículo en las actas de la conferencia en Acceso Abierto (Open Access) en diversos formatos digitales (PDF, HTML, EPUB) e integrarlos en diversas plataformas online como repositorios y bases de datos bajo la licencia CC: Attribution-NonCommercial-ShareAlike 4.0 International (CC BY-NC-SA 4.0) https://creativecommons.org/licenses/by-nc-sa/4.0/.

Ni APANACXVIII ni los editores son responsables ni del contenido ni de las implicaciones de lo expresado en el artículo. 